\title{
Knockdown of CERB expression inhibits proliferation and migration of glioma cells line U251
}

\author{
Zheng KB ${ }^{1}$, Xie ${ }^{1}$, Li YT ${ }^{2}$, Yuan $\mathrm{Y}^{1}$, Wang $\mathrm{Y}^{1}$, Li CH${ }^{1}$, Shi YF ${ }^{1}$ \\ Department of Neurosurgery, Affiliated Hospital of Hebei University, Hebei province, People's Republic of China. \\ syf16882018@163.com
}

\section{ABSTRACT}

BACKGROUND: Glioma is a type of tumor that occurs in the brain and accounts for almost $30 \%$ of all brain and central nervous system tumors and $80 \%$ of all malignant brain tumors. In this study, we investigate the role of CAMP response element-binding protein (CREB) in the progression of glioma.

METHODS: Tissue samples from glioma patients were collected and examined for expression of CREB and its correlation with tumor grades. CREB was then knocked down via siRNA to see if reduced expression of CREB affects cell proliferation and migration. Factors involved in cell cycles, adhesion and apoptosis were examined as well. Moreover, CRESP/CAS9 mediated knockout of CREB was conducted and athymic Nude mice model was used to investigate CREB's role in vivo.

RESULTS: The evaluated expression level of CREB in glioma patients was correlated with tumor grades. Knockdown of CREB via siRNA in glioma cell line U251 significantly inhibited the proliferation and migration of tumor cells. Moreover, CyclinD1 and Bcl-2 expression were reduced, as well as phosphorylation of IRK1/2 and AKT. Additionally, knockout of CREB via CRESP/CAS9 inhibited tumor formation of U251 cells in athymic Nude mice model.

CONCLUSIONS: In conclusion, our data suggest that over expression of CREB may contribute to progression of glioma and knockdown of CREB expression may serve as a novel target for therapy (Tab. 1, Fig. 6, Ref. 25). Text in PDF www.elis.sk.

KEY WORDS: glioma, CREB, cell proliferation, tumor invasion.

\section{Introduction}

Glioma is a type of tumor that occurs in the brain or spine which arises from glial cells (1). The most common and most aggressive subtype of malignant gliomas is glioblastoma (4). Data suggested that hereditary genetic disorders such as neurofibromatoses (type 1 and type 2) and tuberous sclerosis complex are known as factors involved their development (9). Currently, the standard treatment for glioblastoma is the maximal surgical resection combined with radiotherapy and temozolomide $(6,7)$. There is an urgent need to explore novel approaches, such as immunotherapy, to improve outcomes for these patients. Therefore, a better understanding of the molecular mechanism and other prognosis

${ }^{1}$ Department of Neurosurgery, Affiliated Hospital of Hebei University, Hebei province, People's Republic of China, and ${ }^{2}$ Medical College of Hebei University, Hebei province, People's Republic of China

Address for correspondence: YF Shi, No.212 Yuhua East Road, Department of Neurosurgery, Affiliated Hospital of Hebei University, Baoding 071000, Hebei province, People's Republic of China.

Phonel/Fax: +86.0312.5981202

Acknowledgement: This work was supported by grants from Science Foundation of Hebei Province high-level talents support project (Grant No. A2016002082), and Science Foundation of Health and Family Planning Commission of Hebei University (Grant No. 20170817) and Science Foundation of Hebei University (Grant No. 2015Z2003) markers of gliomagenesis will benefit the development of novel targeted therapies for this lethal disease.

A recent report focused on the identification of dysregulated MicroRNAs in glioma demonstrated that miR-433-3p and miR433-5p expression was low in glioma tissues and cell lines and bioinformatics combined with experiment assay suggested that cAMP response element-binding protein(CREB) is a direct target of miR-433-3p (11). In this study, we further investigated the expression of endogenous CREB level from surgery tissue samples obtained from glioma patients.

\section{Material and methods}

\section{Patients and ethic statements}

All patients involved in this study were formally informed for the purpose of using their sample and a letter of consent were signed by everyone who participated. This study was approved by the Ethical Committee of Hebei University. The study methodologies conformed to the standards set by the Declaration of Helsinki. To minimize the influence of general information on the final result, we had conducted a systematic analysis for the gender and ages for these patients. However, no significant difference were observed.

All the glioma samples were collected from the 60 patients who underwent routine surgery at the second hospital affiliated to Hebei University from January 2011 to December 2012. All pa- 


\section{9-315}

tients were confirmed with glioma by pathological examination. Grading of glioma was subjected to World Health Organization (WHO) grading system for astrocytoma, under which tumors are graded from I (least advanced disease - best prognosis) to IV (most advanced disease - worst prognosis). All enrolled patients did not received chemotherapy before the surgery. The healthy brain tissue samples obtained from 10 cases receiving craniocerebral injury decompression were included as control.

\section{Immunohistochemistry analysis (IHC)}

The expression level of CREB proteins from tumor tissues and healthy tissue were detected by immunohistochemistry method. Briefly, the paraffin sections were subsequently deparaffinized with xylene and rehydrated in decreasing concentrations of ethanol. Antigen retrieval was performed by heating sections in an antigen retrieval buffer $(\mathrm{pH}, 6.0)$ in a microwave cooker at $90{ }^{\circ} \mathrm{C}$ for $45 \mathrm{~min}$. After antigen retrieval, endogenous peroxidase activity was blocked via adding $3 \% \mathrm{H}_{2} \mathrm{O}_{2}$ for $10 \mathrm{~min}$. To reduce background, the section was also blocked by normal goat serum (Sigma-Aldrich, St. Louis, MO, USA) for $30 \mathrm{~min}$ at room temperature (RT). Then, the sections were incubated with (same dilution applied for all primary antibodies) rabbit anti-CREB polyclonal antibody (Abcam, Cambridge, MA, USA), overnight at $4{ }^{\circ} \mathrm{C}$. After primary antibody incubation, the sections were washed with PBS and incubated with horseradish peroxidase (HRP) labeled anti-rabbit (Sigma-Aldrich) antibodies (1:100 dilution) at $37^{\circ} \mathrm{C}$ for $60 \mathrm{~min}$ and subsequently washed three times with PBS. The specific reaction between primary antibodies with its targets was visualized by with DAB kit (Sigma-Aldrich) and the section was counterstained with hematoxylin (Sigma- Aldrich) and visualized in Olympus CX22 microscope.

Scoring of IHC results was conducted as follows. Briefly, 5 fields were randomly selected from each section for counting the percentage of CREB positively stained cells. Less than $5 \%$ positively stained was set as 0 score, $6-25 \%$ positively stained was set as score $1.26-50 \%$ positively stained was set as 2 . More than $50 \%$ positively stained was set as score 3 . The staining score of each positive cell was further divided to 0 (no stain), 1 (yellow stain), 2 (tan staining) and 3 (dark tan staining). Multiplication results of CREB positive stained cells score and staining score was used to classify the final grading of CREB positive level for each section. Samples with final score less than 3 were considered as CREB negative (-). Samples with final score equal or higher than 3 were considered as positive. Samples with final score higher than 4 were considered as strongly positive $(++)$.

\section{Cell lines and chemicals}

Human U251 Glioma Cell was purchased from ThermoFisher Scientific and maintained in Dulbecco's Modified Eagle Medium (DMEM, Gibco, Carlsbad, CA, USA) supplemented with $10 \%$ fetal bovine serum (Gibco). The CREB knockout U251 cell was generated by GeneCopoeia Co. Ltd (Guangzhou, China).

\section{Cell proliferation assay (MTT)}

The trypsinized cells were first stained with trypan blue (Sigma-Aldrich) for counting of living cells. Then the single cell suspension was seeded in 96 well plates with a density of $2 \times 10^{4}$ cell in each well. After overnight incubation, indicated treatments were conducted, then the cell proliferation was determined at the indicated time points by using MTS Cell Proliferation Colorimetric Assay kit (Biovision, Milpitas, CA, USA) according to manufacturer's instruction with VICTOR ${ }^{\mathrm{TM}} \mathrm{X} 5$ Multilabel Plate Reader(PerkinElmer, Waltham, MA, USA).

\section{Western blot analysis}

Tissue samples from patients was frizzed by liquid nitrogen for homogenization. Then homogenized tissue samples or cultured cells were lysed by the Laemmli Sample Buffer as previously described $(12,13)$. Proteins from lysate were separated by sodium dodecyl sulfate-polyacrylamide gel electrophoresis (SDS-PAGE) and analyzed by Western blot as previously described (13). Briefly, SDS-PAGE were used for separating denatured proteins and then transferred onto PVDF membrane first. Then, the transferred membranes were probed with rabbit anti-CREB antibody (Abcam), rabbit anti-CyclinD1 antibody (Abcam), rabbit anti- E-cadherin antibody, anti-Bcl-2 monoclonal antibody (Santa Cruz Biotechnology, Santa Cruz, CA, USA), anti-Bcl-2 monoclonal antibody(Santa Cruz), rabbit anti-pERK antibody (Abcam) and rabbit anti-phospho-Akt (Ser473) antibody (Cell signaling technology, Trask Lane Danvers, MA, USA). Specific reactions were detected by using goat anti-rabbit IgG or anti-mouse IgG conjugated with horseradish peroxidase (Sigma-Aldrich) and revealed by a chemiluminescence substrate (Bio-Rad Laboratories, Hercules, CA, USA). All membranes were also probed with GAPDH (Sigma-Aldrich) to normalize the protein loading. The chemiluminescence signal was recorded by the ChemiDoc XRS imaging system (Bio-Rad Laboratories) and all data analyses were conducted by the ImageLab Program (Bio-Rad Laboratories).

\section{Reverse transcription and Real-Time PCR ( $q P C R$ )}

Total cellular RNA from tissue sample or cell line was isolated by using TRIzol Reagent (Invitrogen, Carlsbad, CA, USA) according to manufacturer's instruction. RNase-free DNase (Promega, Madison, Wisconsin, USA) was added to remove genome DNA contamination from the RNA isolation procedure. Reverse transcription was performed by AMV reverse transcriptase (Promega) using a combination of oligo dT and random hexamer according to manufacturer's recommendation. Real-time PCR detection with SYBR Green Mix (Life technologies) for the targeted genes was previously described $(12,14)$. Transcript of GAPDH was detected from the same samples to serve as housekeeping gene for normalization of RNA loading. Gene expression was quantified by $2^{-\Delta \Delta C T}$ method as previously described (15). Primers used in this study are listed in Table 1.

\section{Transwell cell invasion assay}

To evaluate the capability of cell invasion, the Transwell cell invasion assay was conducted as previously described with modifications (16). Briefly, 12 hours before the experiment, the cell culture medium was discarded and replaced with FBS free DMEM for miRNA transfection. 24 hours after transfection, the cell was 
Tab. 1. Scoring and statistical analysis for CREB expression from glioma patients.

\begin{tabular}{lccccc}
\hline \multirow{2}{*}{ Grade } & $\mathrm{n}$ & \multicolumn{3}{c}{ CREB } & \multirow{2}{*}{ Positive rate (\%) } \\
\cline { 3 - 5 } & & - & + & ++ & \\
\hline Control & 5 & 3 & 2 & 0 & 40 \\
Grade I-II & 15 & 5 & 10 & 0 & $66.7^{\#}$ \\
Grade III & 12 & 1 & 7 & 4 & $91.7^{\# *}$ \\
Grade IV & 28 & 0 & 9 & 19 & $100^{\# \Delta}$ \\
\hline
\end{tabular}

trypsinized and stained with trypan blue for cell counting. In total 100uL cell suspension medium was added into Transwell chamber and cultured for another $48 \mathrm{~h}$. Then those chambers were stained with hematoxylin. A total of six microscopic fields were randomly selected from each chamber and were captured for quantification.

\section{Statistical analysis.}

Statistical analyses were performed using SPSS 13.0 (SPSS Inc.). All data are presented as Mean \pm SD. Differences in indicators between samples were subjected to the Student's t test. A two tailed $\mathrm{p}$ value of less than 0.05 was considered statistically significant.

\section{Results}

CREB over expression in glioma patients was correlated with glioma grade

CREB (cAMP response element-binding protein) is a cellular transcription factor which binds to cAMP response elements (CRE) to increase or decrease the transcription of the downstream genes (17). A recent study demonstrated that miR-433-3p and miR433-5p have low expression in glioma tissues and cell lines and bioinformatics combined with experimental assay suggested that
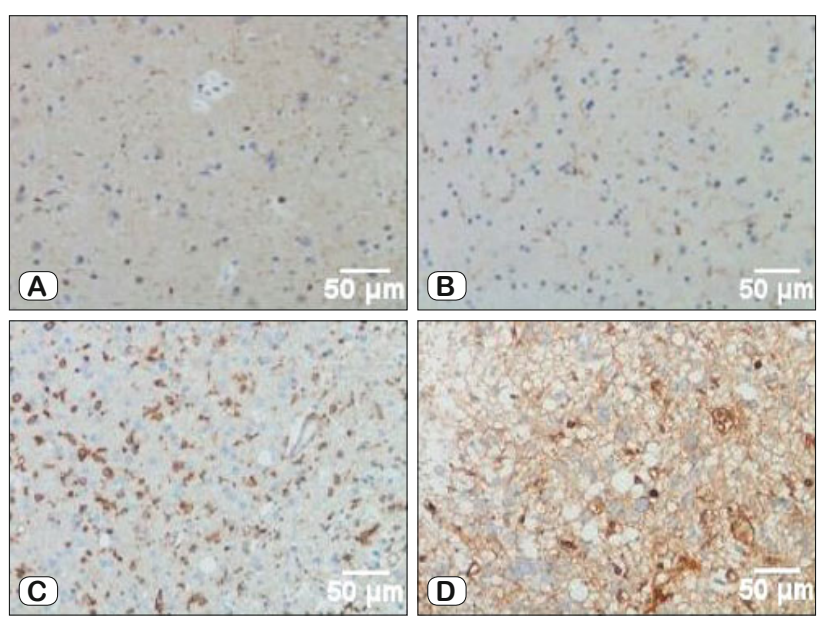

Fig. 1. Immunohistochemistry analysis of CREB expression in samples from healthy controls and glioma patients. A: Representative IHC image of CREB expression in sample from healthy individuals; B: Representative IHC image of CREB expression in sample from Grade I to II glioma patients; C: Representative IHC image of CREB expression in sample from Grade III glioma patients; D: Representative IHC image of CREB expression in sample from Grade IV glioma patients.
cAMP response element-binding protein(CREB) is a direct target of miR-433-3p (11). It is interesting to know if CREB is over expressed in glioma patients and what kind of role CREB plays for the prognosis of glioma. To test our hypothesis, we first examined the expression of CREB in tumor samples obtained from patients. Based on our IHC analysis, CREB expression in healthy samples is almost non-detectable or expressed at very low level (Fig. 1A). In samples obtained from Grade I to II glioma patients, CREB expression level is similar to healthy controls (Fig. 1B), however, CREB expression level was significantly higher in samples obtained from Grade III to IV glioma patients (Figs 1C and 1D).

To confirm our observation for increased CREB expression in glioma patients, we further analyzed the CREB mRNA and protein level via real-time PCR (qPCR) and western blotting. It was demonstrated that increased CREB expression level was correlated with grade of glioma obtained from patient's sample. There were nearly 3 folds and 4 folds changes in CREB mRNA in patients with grade III and grade IV glioma, respectively (Fig. 2A). Western blot also confirmed the observation from qPCR (Fig. 2B). Moreover, quantification of WB data (CREB/GAPDH ratio)

(A)

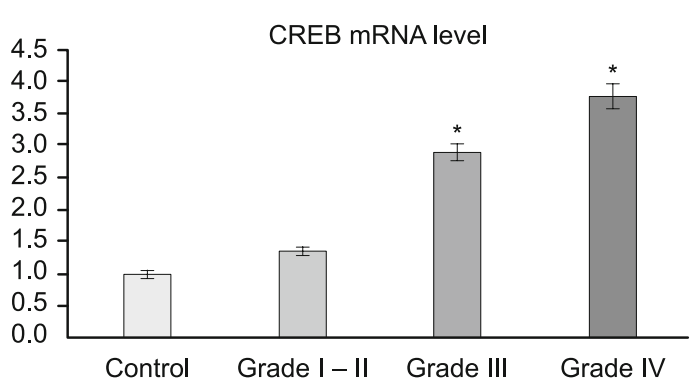

(B) CREB GAPDH

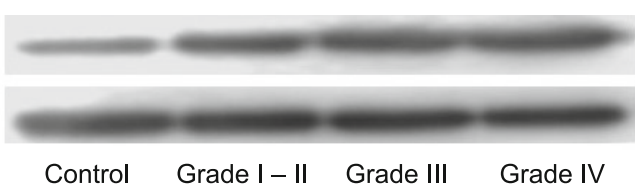

C

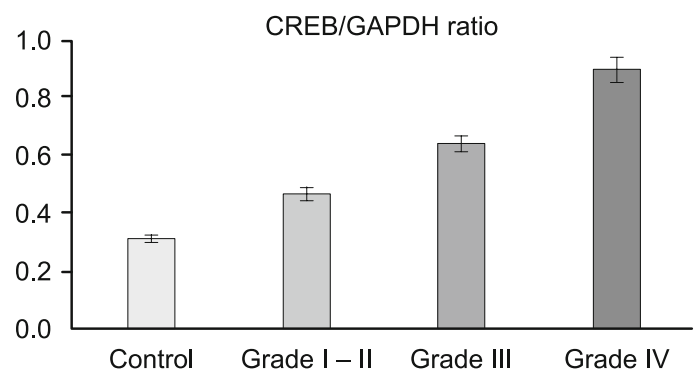

Fig. 2. Evaluation of CREB expression in samples from healthy controls and glioma patients via qPCR and WB, A: CREB mRNA level in samples obtained from healthy individuals and glioma patients with different Grades; A: CREB protein level examined by WB in samples obtained from healthy individuals and glioma patients with different Grades; C: Quantification of WB data from healthy individuals and glioma patients with different Grades. 
309-315
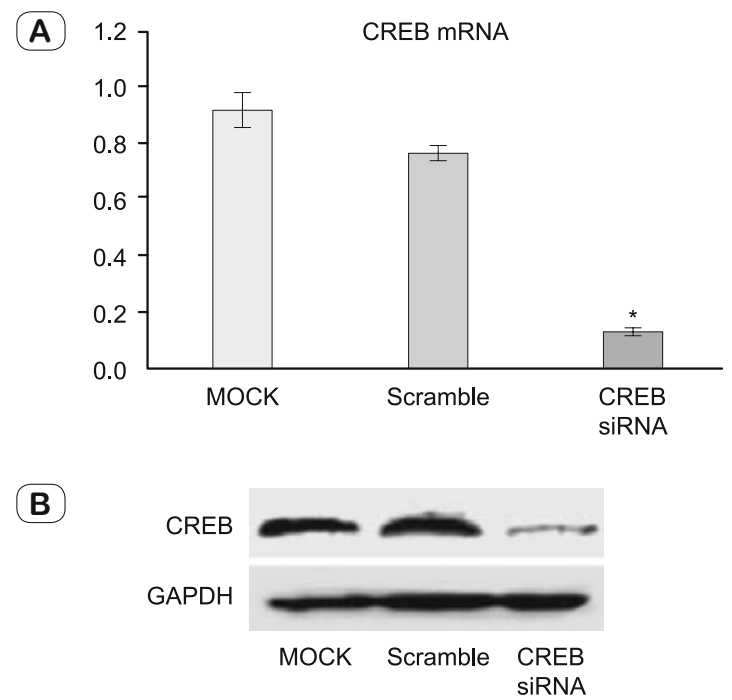

Fig. 3. Knockdown of CREB expression via siRNA in glioma cell line U251, A: Evaluation of CREB mRNA level in U251 cells without transfection or transfected with scramble control siRNA and CREB siRNA, B: WB evaluation of CREB protein level in U251 cells without transfection or transfected with scramble control siRNA and CREB siRNA.

suggested a similar trend as we observed in qPCR. The detailed data of CREB expression analysis for every patient are listed in Table.1. Taken together, these data suggest that CREB expression is evaluated in glioma patients and correlates with grade of glioma.

(A)

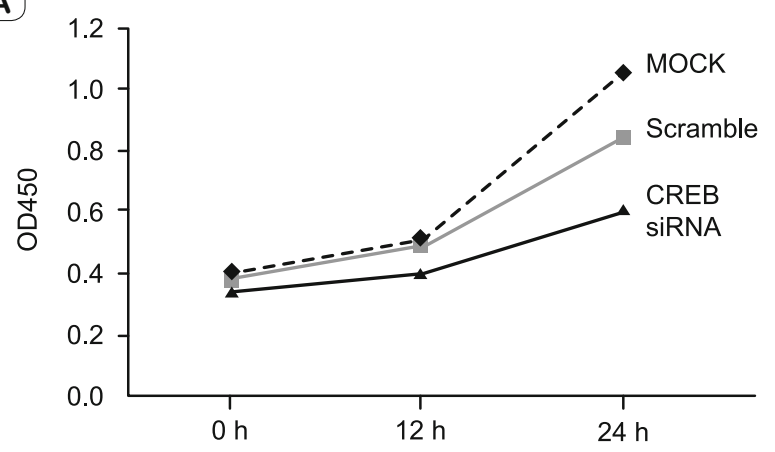

Knock-down of CREB via siRNA inhibits glioma cells growth and cell migration

To further explore the role CREB expression plays in the prognosis of glioma, we knocked-down the expression via siRNA in glioma derived cell lines. The siRNA targeted CREB was transfected to U251 cell alone with its scramble control cells. The expression of both mRNA or protein of CREB was further tested via $\mathrm{qPCR}$ and western blot. Based on our results, transfection of CREB-specific siRNA led to $80 \%$ reduction of CREB mRNA level which is similar to Western Blot data (Figure.2A and B), which suggested the siRNA mediated CREB knock-down is highly efficient.

After confirmation of CREB knock-down efficiency in U251 cell, the proliferation of U251 cell was tested first. On the one hand, based on MTT cell proliferation assay, U251 cells with transfection of scramble siRNA demonstrated very similar proliferation rate as normal U251 cells without any treatment (Fig. 3A). However, U251 cells transfected with CREB specific siRNA demonstrated reduced proliferation level within 24 hours posted transfection. OD450 value demonstrated that proliferation of U251 cells transfected with CREB specific siRNA only showed $50 \%$ and $80 \%$ proliferation rate compared with control cells or cells transfected with control siRNA after 12 hours and 24 hours, respectively. On the other hand, the Traswell Cell migration assay was also conducted as well to test if the reduction of cell proliferation could lead to reduced cell migration. The representative images of each groups are shown in Figure 3B. As shown in these images, knock-down expression of CREB significantly reduced the cell migration. Further quantification demonstrated that there was
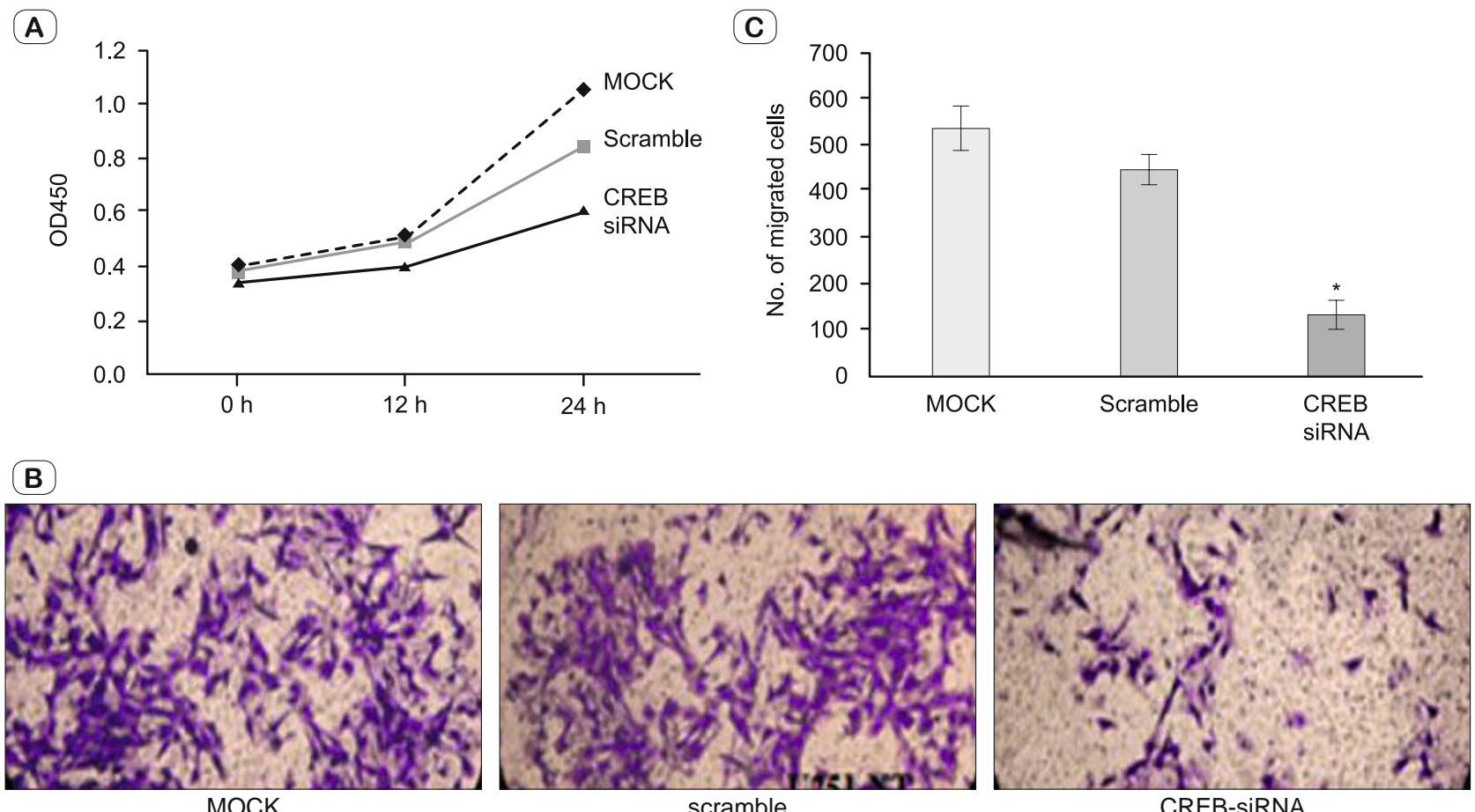

Fig. 4. Knockdown of CREB expression in U251 inhibits proliferation and cell migration, A: Cell proliferation assay for U251 cells without transfection or transfected with scramble control siRNA and CREB siRNA; B: Representative image of cell migratin assay of U251 cell without transfection or transfected with scramble control siRNA and CREB siRNA. 


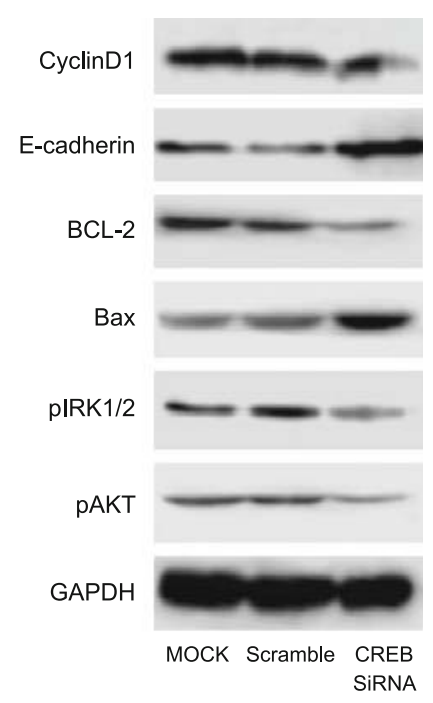

Fig. 5. Knockdown of CREB expression in U251 changing the expression of host factor involved in cell cycle and apoptosis, WB blot analysis for CyclinD1, Bcl-2, Bax, phosphorylated IRK1/2 and phosphorylated AKT, E-cadherin.

A

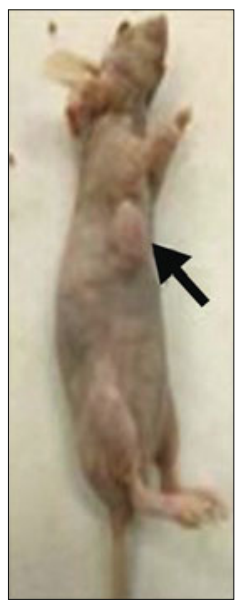

U251

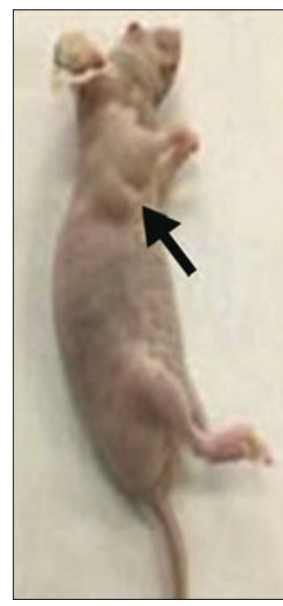

U251-NT
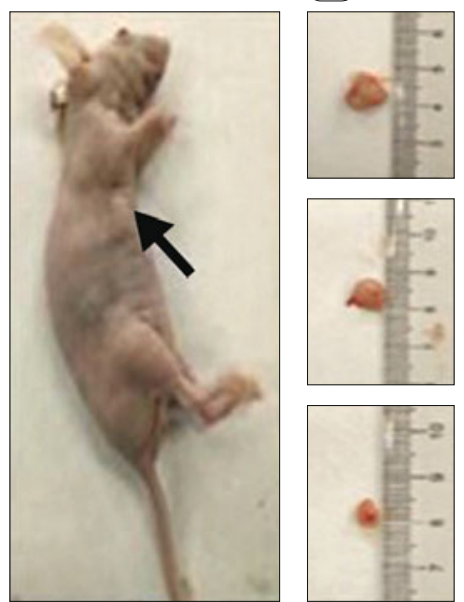

U251-KO a near $70 \%$ reduction of cell migration if CREB specific siRNA was introduced into the cells (Fig. 3C). Taken together, these data suggested that knock-down of CREB via siRNA inhibited glioma cells growth and cell migration.

Knock-down of CREB affects expression of proteins involved in cell proliferation, apoptosis and adhesion

To underline the mechanism of CREB expression played during proliferation of glioma cells, we also examined the expression level of other cell cycles or apoptosis related proteins such as $\mathrm{Cy}$ clinD1, BCL-2 and other genes. Based on our data, CyclinD1, Bcl2, phosphorylated IRK $1 / 2$ and phosphorylated AKT were shown to be reduced in CREB-specific siRNA transfected cell, while Ecadherin and Bax were increased (Fig. 5). Taken together, these data suggest that knock-down of CREB is able to inhibit proliferation of glioma derived cell lines in vitro.

Knockout of CREB via CRESP/CAS9 inhibits glioma cells line growth in vivo

Based on the in vitro study, we concluded that knockdown of CREB in U251 cells is able to inhibit proliferation and migration

\section{(B)}
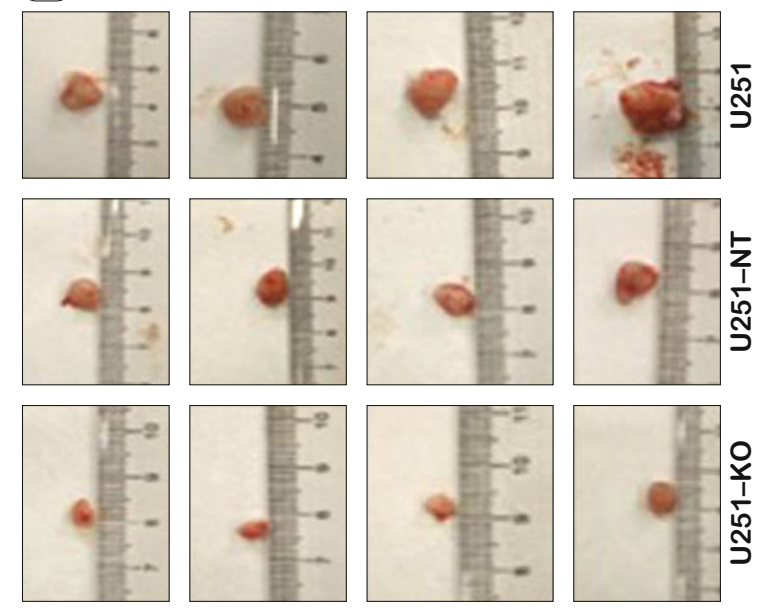

(C)

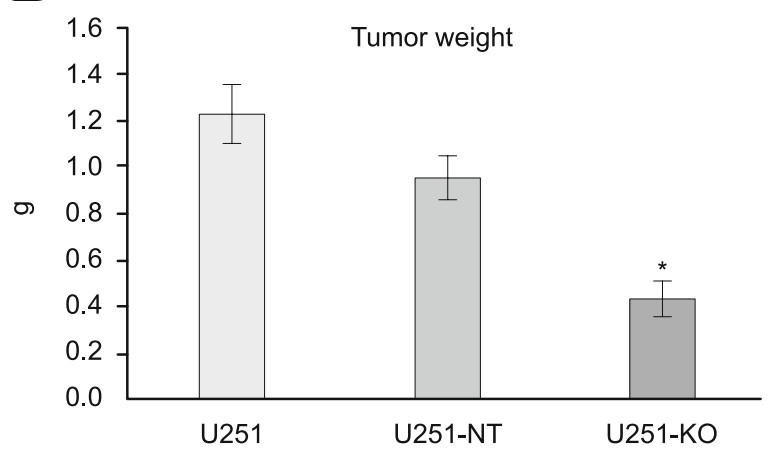

Fig. 6. Knockout of CREB via CRESP/CAS9 inhibits glioma cells line growth in vivo, A. Representative image of tumor growth in NOD mice injected with normal U251 cell, U251-NT, U251KO; B: evaluated for tumor size obtained from NOD mice injected with normal U251 cell, U251NT, U251KO; C: Quantification of tumor weight obtained from NOD mice injected with normal U251 cell, U251-NT, U251KO. 


\section{9-315}

of tumor cell, however, it is still unclear if knockdown of CREB could function similarly in vivo (Fig. 4). To confirm our speculation, we generated CREB knockout cell line via CRISPR-Cas9 mediated genome editing (conducted by GeneCopoeia Co. Ltd) and injected the knockout cell line to nod mice. A control cell line (U251-NT) was used as control. The formation of tumor and tumor size was significantly higher in nod mice injected with normal U251 cell and U251-NT control cell than in mice injected with CREB knockout (KO) cells (Figs 6A and B). To quantitatively compare the formation of tumor, we evaluated the tumor weight from mice injected with different cells as well and it consisted of direct observation of small size of CERB-KO U251 cell. Taken together, our data suggested that knockout CREB via CRESP/ CAS9 inhibits glioma cells line growth in vivo.

\section{Discussion}

CREB (cAMP response element-binding protein) is a cellular transcription factor or co-activator which binds to certain DNA sequences called cAMP response elements (CRE), thereby increasing or decreasing the transcription of the downstream genes (18). CREB contains a basic/leucine zipper structure (bZIP) and can be activated through phosphorylation at Ser-133 via a number of kinases pathways, including the PKA, PKC, CaM kinases, p90RSK, and ERK1/2 signaling pathways $(19,20)$. CREB has been indicated to play key roles in the development and progression of multiple cancers, including malignant mesothelioma, non-small cell lung cancer, leukemia and breast cancer (21-23). In our study, we first tested if CREB expression is evaluated in glioma samples obtained from patients. It appears that CREB expression level is correlated with the Grade III to IV glioma patients, which is consistent with previous observation of reduced expression of miR-433-3p and miR-433-5p (potential regulator for CREB) was correlated with evaluated CREB level in glioma patients.

In this study, we investigated the role of CREB in glioma cell line both in vitro and in vivo. On the one hand, in vitro study conducted in U251 cell suggested that knockdown of CREB expression significantly inhibited proliferation and migration of glioma cell lines. On the other hand, in vivo nod mice model further confirmed that knockout of CREB via CRESP/CAS9 technology prevented tumor formation in vivo.

It has been suggested that CREB was capable to regulate glioblastoma tumor cell proliferation which involves activities downstream from both the MAPK and PI3K pathways and modulate the expression of three key cell cycle factors, such as cyclin B1, cyclin D1 and PCNA (24). Therefore, in our experiment, we also tested the expression of cell cycle factors as well. Our data suggested that knockdown of CREB in glioma cell lines reduce the expression of CyclinD1 as well as reduced phosphorylated IRK1/2 and AKT, which is consisted with previous reports. Moreover, besides reduced expression of factors involved in cells cycles, we also notice that expression of genes involved in apoptosis was also changed, such as reduction of anti-apoptotic protein Bcl-2 and pro-apoptotic protein Bax (Figure.5), which may contribute to anti-proliferation effect in CREB knock down cells.
In our experiment, we also notice that knockdown of CREB expression in glioma cell line U251 inhibits cell migration. It has been suggested that the E-cadherin-catenin complex plays a key role in cellular adhesion and loss of E-cadherin's function is thought to contribute to progression in cancer by increasing proliferation, invasion, and/or metastasis in many different tumor types (25). Our data suggested that knock down of CREB will lead to evaluation of E-cadherin, which may contribute to cell migration inhibition as E-cadherin plays a key role in cellular adhesion. Moreover, evaluation of E-cadherin may inhibit proliferation of U251 cell as well. In conclusion, our data suggest that over expression of CREB may contribute to progression of glioma and knockdown of CREB expression may serve as a novel target for therapy.

\section{Reference}

1. Mamelak AN, Jacoby DB. Targeted delivery of antitumoral therapy to glioma and other malignancies with synthetic chlorotoxin (TM-601). Expert Opin Drug Deliv 2007; 4: 175-186.

2. Goodenberger ML, Jenkins RB. Genetics of adult glioma. Cancer Genet 2012; 205: 613-621.

3. Van Meir EG, Hadjipanayis CG, Norden AD, Shu HK, Wen PY, Olson JJ. Exciting new advances in neuro-oncology: the avenue to a cure for malignant glioma. CA Cancer J Clin 2010; 60: 166-193.

4. Filley AC, Dey M. Dendritic cell based vaccination strategy: an evolving paradigm. J Neurooncol 2017.

5. Stupp R, Hegi ME, Mason WP, van den Bent MJ, Taphoorn MJ, Janzer RC, Ludwin SK, Allgeier A, Fisher B, Belanger K, Hau P, Brandes AA, Gijtenbeek J, Marosi C, Vecht CJ, Mokhtari K, Wesseling P, Villa S, Eisenhauer E, Gorlia T, Weller M, Lacombe D, Cairncross JG, Mirimanoff RO. Effects of radiotherapy with concomitant and adjuvant temozolomide versus radiotherapy alone on survival in glioblastoma in a randomised phase III study: 5-year analysis of the EORTC-NCIC trial. Lancet Oncol 2009; 10: 459-466.

6. Minniti G, De Sanctis V, Muni R, Filippone F, Bozzao A, Valeriani M, Osti MF, De Paula U, Lanzetta G, Tombolini V, Maurizi Enrici R. Radiotherapy plus concomitant and adjuvant temozolomide for glioblastoma in elderly patients. J Neurooncol 2008; 88: 97-103.

7. Komotar RJ, Otten ML, Moise G, Connolly ES, Jr. Radiotherapy plus concomitant and adjuvant temozolomide for glioblastoma-a critical review. Clin Med Oncol 2008; 2: 421-422.

8. Stupp R, Mason WP, van den Bent MJ, Weller M, Fisher B, Taphoorn MJ, Belanger K, Brandes AA, Marosi C, Bogdahn U, Curschmann J, Janzer RC, Ludwin SK, Gorlia T, Allgeier A, Lacombe D, Cairncross JG, Eisenhauer E, Mirimanoff RO. Radiotherapy plus concomitant and adjuvant temozolomide for glioblastoma. N Engl J Med 2005; 352: 987-996.

9. Reuss D, von Deimling A. Hereditary tumor syndromes and gliomas. Recent Results Cancer Res 2009; 171: 83-102.

10. Fukushima S, Narita Y, Miyakita Y, Ohno M, Takizawa T, Takusagawa Y, Mori M, Ichimura K, Tsuda H, Shibui S. A case of more than 20 years survival with glioblastoma, and development of cavernous angioma as a delayed complication of radiotherapy. Neuropathology 2013; 33: 576-581. 
11. Sun S, Wang X, Xu X, Di H, Du J, Xu B, Wang Q, Wang J. MiR433-3p suppresses cell growth and enhances chemosensitivity by targeting CREB in human glioma. Oncotarget 2017; 8: 5057-5068.

12. Patel D, Nan Y, Shen M, Ritthipichai K, Zhu X, Zhang YJ. Porcine reproductive and respiratory syndrome virus inhibits type I interferon signaling by blocking STAT1/STAT2 nuclear translocation. J Virol 2010; 84: $11045-11055$.

13. Nan Y, Wang R, Shen M, Faaberg KS, Samal SK, Zhang YJ. Induction of type I interferons by a novel porcine reproductive and respiratory syndrome virus isolate. Virology 2012; 432: 261-270.

14. Patel D, Opriessnig T, Stein DA, Halbur PG, Meng XJ, Iversen PL, Zhang YJ. Peptide-conjugated morpholino oligomers inhibit porcine reproductive and respiratory syndrome virus replication. Antiviral Res 2008; 77: 95-107.

15. Livak KJ, Schmittgen TD. Analysis of relative gene expression data using real-time quantitative PCR and the 2(-Delta Delta C(T)) Method. Methods 2001; 25: 402-408.

16. Marshall J. Transwell (R) invasion assays. Methods Mol Biol 2011; 769: 97-110.

17. Bourtchuladze R, Frenguelli B, Blendy J, Cioffi D, Schutz G, Silva AJ. Deficient long-term memory in mice with a targeted mutation of the cAMP-responsive element-binding protein. Cell 1994; 79: 59-68.

18. Montminy MR, Bilezikjian LM. Binding of a nuclear protein to the cyclic-AMP response element of the somatostatin gene. Nature 1987; 328: $175-178$.
19. Yamamoto KK, Gonzalez GA, Biggs WH, 3rd, Montminy MR. Phosphorylation-induced binding and transcriptional efficacy of nuclear factor CREB. Nature 1988; 334: 494-498.

20. Shaywitz AJ, Greenberg ME. CREB: a stimulus-induced transcription factor activated by a diverse array of extracellular signals. Annu Rev Biochem 1999; 68: 821-861.

21. Aggarwal S, Kim SW, Ryu SH, Chung WC, Koo JS. Growth suppression of lung cancer cells by targeting cyclic AMP response elementbinding protein. Cancer Res 2008; 68: 981-988.

22. Shankar DB, Sakamoto KM. The role of cyclic-AMP binding protein (CREB) in leukemia cell proliferation and acute leukemias. Leuk Lymphoma 2004; 45: 265-270.

23. Park JK, Park SH, So K, Bae IH, Yoo YD, Um HD. ICAM-3 enhances the migratory and invasive potential of human non-small cell lung cancer cells by inducing MMP-2 and MMP-9 via Akt and CREB. Int J Oncol 2010; 36: 181-192.

24. Daniel P, Filiz G, Brown DV, Hollande F, Gonzales M, D'Abaco G, Papalexis N, Phillips WA, Malaterre J, Ramsay RG, Mantamadiotis T. Selective CREB-dependent cyclin expression mediated by the PI3K and MAPK pathways supports glioma cell proliferation. Oncogenesis 2014; 3: e108.

25. Beavon IR. The E-cadherin-catenin complex in tumour metastasis: structure, function and regulation. Eur J Cancer 2000; 36: 1607-1620.

Received December 1, 2018. Accepted January 21, 2019. 